\title{
Short Communication: A Hypothesis for a Physical Therapy to Avoid Covid-19 Patients' Death
}

\section{Giulio Lorenzini}

Department of Engineering and Architecture, University of Parma, Parco Area delle Scienze 181/A, Parma 43124, Italy

Corresponding Author Email: giulio.lorenzini@unipr.it

https://doi.org/10.18280/ijdne.150301

Received: 20 April 2020

Accepted: 1 June 2020

\section{Keywords:}

Covid-19, SARSCOV-2, physical therapy, death prevention

\begin{abstract}
In this Short Communication, it is presented a hypothesis of therapy, which could reveal useful to treat Covid-19 patients, especially those in a very serious condition, like in "phase 3" of the disease. The therapy presented is not based on a pharmaceutical approach but just on a physical one. The therapy itself is not considered here to be a way to fully recover from Covid-19, but as a technique to avoid the patients' death, thus making of Covid-19 a non-lethal disease. Being a hypothesis, even if extremely rational, the therapy proposed will, nevertheless, need a deep and careful experimental verification before it can be applied to humans.
\end{abstract}

\section{INTRODUCTION}

A few important preconditions underlie the hypothesis here presented:

1) it is well known that, to disinfect medical devices or in the field of food preservation, UV-C lamps are employed (with a wave length of about $270 \mathrm{~nm}$ ). They have widely proved their ability in killing germs, bacteria and viruses of any kind, nevertheless having a short radius of action, thus acting just if the source acts almost in contact with the surface to purify.

2) UV-C rays could, consequently, be able to kill also the SARSCOV-2 virus, responsible of Covid-19.

3) It is well known by the International Scientific Community that the virus SARSCOV-2 attacks many organs of the patient's body, but its more evident and dangerous effects are on the lungs, where it may cause an interstitial pneumonia.

4) The main cause of death, probably the only one, in the Covid-19 patients is for sure due to the breathing complications consequent to the afore said interstitial pneumonia.

5) From a recent American research [1] it can be evicted that the "far UV-C" rays, with a wavelength varying between 207 and $222 \mathrm{~nm}$, have the same ability to kill germs, bacteria and viruses, as seen in point 1 , but they have a significantly reduced biological impact on the sane human tissues.

6) The "far UV-C" rays do not have penetrating power in the human skin and thus an external source to the body of the patient could not help purifying the lungs.

\section{THE THERAPEUTIC HYPOTHESIS}

In the cases of Covid-19 where the disease has heavily attacked the lungs (especially in "phase 3" of the disease), with breathing complications and/or insurgence of interstitial pneumonia, we could plan the introduction, in the body of the patient, of a "far UV-C" rays probe, proceeding from the throat and reaching the lungs via a technique of "intubation" which is well known to Anesthetist M. Doctors. Moreover, with the modern LED technologies, such probes could be realised of various dimensions (suggested by the M. Doctors), shape (presumably cylindrical) and material consistence (presumably not-rigid). Once the probe has been located as close as possible to the lungs, it would be switched on the emission of "far UV-C" rays, to act on the pulmonary region, and to cancel or at least reduce the viral charge present there. In any case, this could be applied to a patient just after having reckoned in laboratory the least time of exposition to the "far UV-C" rays which is necessary to kill completely the SARSCOV-2 or anyway to reduce its presence to a percentage enough low to significantly help the patient's Immune System and the other medical therapies to act efficiently and save a life.

\section{FAQ}

1) The Interstitial Pneumonia implies the presence of the SARSCOV-2 in the lungs? The SARSCOV-2 is present in many organs of the patient's body, but it is in the lungs that it gives the "worst results", often generating an Interstitial Pneumonia which damages the alveolus cells, limiting breathing, first partially and then, in the worst cases, until asphyxiation. Technically speaking [2]: Interstitial Pneumonia implies a diffused alveolar damage, causing an oedema, diffused and important, of the alveolar septa, while symptoms imply dyspnoea, generally worsening in 7-10 days and reaching Respiratory Distress.

2) As told in point 6 of Section 1, the "far UV-C" rays [3] cannot be applied externally to the patient's body as they do not penetrate the physical barrier of skin: wouldn't the probe have the same problem, if not located in direct contact with the virus-infected cells? In other words: would all the infected cells, within the lungs, be reached by the probe's 
effects, even if not in "perfect" contact with them? The "far UV-C" rays do not penetrate skin, which is a compact structure. But if such rays are emitted, as proposed, near the lungs, which are made of a "porous" structure, they will not have difficulties to cross them.

3) The SARSCOV-2 virus could shift inside mucosas and in the capillaries, thus maybe (but this should be tested) not being affected /killed by the "far UV-C" rays. Is this a limit to the therapy proposed? I can reply "no", as the main risk for the Covid-19 patient, especially in an advanced stage, is the connection arising between the virus and the alveoluses, which basically limits breathing until forbidding it completely, thus leading to death. It is absolutely plausible that the "far UV-C" rays are able to destroy such connection, avoiding thus asphyxiation and the patient's death. Nevertheless it has to be clearly highlighted that the "far UV-C" rays won't be able to remove the WHOLE viral charge inside the patient's body but, I reckon, it would yet be an excellent result if we could move away the risk of an imminent death, so giving M. Doctors enough time to go on treating the patient with "traditional" therapies.

4) If, in general, UV-C rays can damage the virus cells, they could also damage the human body cells. May this happen? Sure: UV-C rays are not healthy for human body, and there is no doubt about this. But it has to be highlighted that the body cells dimensions are much bigger than the viral cells ones: consequently, it is undeniable that the "damage" caused to the virus by UV-C rays is extremely bigger than that caused to the healthy cells. Moreover, as specified above, we would employ "far UV-C" rays (lower wavelength), who have proved (see point 5 in Section 1) to be less harmful to human tissues. Nevertheless, laboratory tests should carefully verify which is the smallest time of exposition to "far UV-C" rays allowing for killing SARSCOV-2 virus and for damaging the least possible the healthy cells. Anyway, if I am allowed to make a hyperbole, as in an oncologic patient there is often the case to use radiotherapy, which is certainly not "ideal" for the healthy cells of the patient, it is preferred to attack the diseased cells, to prevent an imminent risk, even if this means accepting a possible limited damage to the heathy cells. Of course, this "example" is evidently an exaggeration, as in oncology the rays utilised are "Gamma" rays, which are not comparably dangerous with respect to "far UV-C" rays! Moreover, still in oncology, diseased cells to be attacked have the same dimensions of healthy cells, while in the present case virus cells are much smaller: it is, thus, reasonable to think that a time of exposition to "far UV-C" rays, long enough to kill the SARSCOV-2 virus, would instead not particularly damage the healthy cells. Naturally this "minimum time" should be carefully pre-tested in laboratory.

\section{CONCLUSIONS}

This Short Communication presents a hypothesis of therapy to treat Covid-19 patients, especially those in "phase 3 " of the disease. The therapy is based on the application of "far UV-C" rays to the patient's lungs, via a suitable probe inserted by "intubation" in the patient's body throat, to be then located as close as possible to the lungs. A few decisive preconditions have been discussed and the therapy has been outlined. The therapy proposed here is not based on a pharmaceutical approach but on a physical one. Not to generate misunderstandings, the aim of this therapy is not to letting a patient fully recovering from Covid-19, but to avoid the his/her death, so transforming Covid-19 in a non-lethal disease. Deep and careful experimental verification will be, anyway, necessary before the therapy described can be directly applied to people.

\section{ACKNOWLEDGMENT}

The author of this Short Communication, Giulio Lorenzini, wishes to thank warmly M. Doctor Manuela Campesato, from Anaesthesia and Pain Therapy Unit "Melotti", Emergency and Urgency Department, St.Orsola-Malpighi General Hospital, Bologna, Italy, for having given the first positive authoritative Medical opinion about the therapy proposed by the author, without which the author himself would not have had enough courage to go ahead proposing the present study!

\section{REFERENCES}

[1] Welch, D., Buonanno, M., Grilj, V., Shuryak, I., Crickmore, C., Bigelow, A.W., Randers-Pehrson, G., Johnson, G.W., Brenner, D.J. (2018). Far-UVC light: A new tool to control the spread of airborne-mediated microbial diseases. Scientific Reports, 8: 2752. https://doi.org/10.1038/s41598-018-21058-w

[2] https://www.msdmanuals.com/itit/professionale/malattie-polmonari/pneumopatieinterstiziali-diffuse/polmonite-interstiziale-acuta

[3] it.wikipedia.org $>/$ wiki/Radiazione-ultravioletta 